\title{
Estimation of the Parameter Covariance Matrix for a One-Compartment Cardiac Perfusion Model Estimated from a Dynamic Sequence Reconstructed Using MAP Iterative Reconstruction Algorithms
}

\author{
Grant T. Gullberg, Ronald H. Huesman, Bryan W. Reutter, Jinyi Qi, and Dilip N. Ghosh Roy
}

\begin{abstract}
In dynamic cardiac SPECT estimates of kinetic parameters of a one-compartment perfusion model are usually obtained in a two step process: 1) first a MAP iterative algorithm, which properly models the Poisson statistics and the physics of the data acquisition, reconstructs a sequence of dynamic reconstructions, 2) then kinetic parameters are estimated from time activity curves generated from the dynamic reconstructions. This paper provides a method for calculating the covariance matrix of the kinetic parameters, which are determined using weighted least squares fitting that incorporates the estimated variance and covariance of the dynamic reconstructions. For each transaxial slice sets of sequential tomographic projections are reconstructed into a sequence of transaxial reconstructions using for each reconstruction in the time sequence an iterative MAP reconstruction to calculate the maximum a priori reconstructed estimate. Time-activity curves for a sum of activity in a blood region inside the left ventricle and a sum in a cardiac tissue region are generated. Also, curves for the variance of the two estimates of the sum and for the covariance between the two ROI estimates are generated as a function of time at convergence using an expression obtained from the fixed-point solution of the statistical error of the reconstruction. A one-compartment model is fit to the tissue activity curves assuming a noisy blood input function to give weighted least squares estimates of blood volume fraction, wash-in and wash-out rate constants specifying the kinetics of ${ }^{99 m} \mathrm{Tc}$-teboroxime for the left ventricular myocardium. Numerical methods are used to calculate the second derivative of the chi-square criterion to obtain estimates of the covariance matrix for the weighted least square parameter estimates. Even though the method requires one matrix inverse for each time interval of tomographic acquisition, efficient estimates of the tissue kinetic parameters in a dynamic cardiac SPECT study can be obtained with present day desk-top computers.
\end{abstract}

\section{INTRODUCTION}

Previously efficient estimates of dynamic cardiac SPECT kinetic parameters of a one-compartment cardiac perfusion model were obtained using weighted least squares estimates of dynamic reconstructions that were obtained from the inversion of the Fisher information matrix [1]-[3]. Each reconstruction in the dynamic sequence was reconstructed using matrix inverse reconstruction. The reconstruction was a solution to a weighted least squares optimization problem, which provided a weighted least squares estimate of the reconstructed values and estimates of the spatial covariances between the reconstructed values. These estimates were used to generate time activity curves for a sum of activity in a blood region inside the left ventricle and a sum in a cardiac tissue region, for the variance of the two estimates of the sum, and for the covariance between the two ROI estimates. The weighted least squares estimates and covariances for the reconstructed time activity curves were fit to a one-compartment perfusion model to obtain weighted least squares estimates of the kinetic model parameters (wash-in, wash-out, and fractional blood volume). It was shown that the weighted least squares estimates of the kinetic model parameters gave lower

This work was supported in part by the National Institutes of Health under Grant RO1-HL50663 and by the Director, Office of Science, Office of Biological and Environmental Research, Medical Sciences Division of the U.S. Department of Energy under contract DE-AC0376 SF00098.

G. T. Gullberg is with the E. O. Lawrence Berkeley National Laboratory, Berkeley, CA 94720 USA.

R. H. Huesman is with the E. O. Lawrence Berkeley National Laboratory, Berkeley, CA 94720 USA.

B. W. Reutter is with the E. O. Lawrence Berkeley National Laboratory, Berkeley, CA 94720 USA.

J. Qi is with the E. O. Lawrence Berkeley National Laboratory, Berkeley, CA 94720 USA and the Department of Biomedical Engineering, University of California, Davis, CA USA.

D. N. Ghosh Roy is with the Department of Radiology, University of Utah, Salt Lake City, CA 84108 USA. 
variance than the unweighted least squares estimates. However, in general the dynamic sequence of reconstructions is obtained using ML [4] or MAP [5] iterative algorithms, which properly model the Poisson statistics and the physics of the data acquisition.

Efficient estimates of dynamic cardiac SPECT kinetic parameters are determined using weighted least squares fitting that incorporates the variance and covariance of maximum a priori estimates of dynamic reconstructions. For one transaxial slice a sequential set of tomographic projections are reconstructed into a sequence of $64 \times 64$ images using for each reconstruction in the time sequence an iterative MAP reconstruction to calculate the maximum $a$ priori reconstructed estimate. Physical factors such as attenuation, geometric response of the collimator and scatter can be included in the projection equations. Time-activity curves for a sum of activity in a blood region inside the left ventricle and a sum in a cardiac tissue region are generated from the attenuation and geometric corrected transaxial reconstructions. The variance of the two estimates of the sum, and the covariance between the two ROI estimates are generated from the inverse of the Fisher information matrix plus a prior term. A two-compartment model is fit to the blood and tissue activity curves to give weighted least squares estimates of blood volume fraction, wash-in and wash-out rate constants specifying teboroxime kinetics for the left ventricular myocardium.

This paper is an updated version of a paper presented at the 2003 IEEE Nuclear Science Symposium and Medical Imaging Conference [6]. The paper investigates the errors in kinetic model parameters for a one-compartment cardiac perfusion model when iterative MAP reconstruction algorithms are used to obtain the dynamic sequence of reconstructions. First the theory is presented for estimating the covariance matrix for reconstructed estimates of a MAP reconstruction and for estimating the covariance matrix for the parameters of a compartment model fit to a sequence of the estimated dynamic reconstructions. Then methods and results are presented for the simulation of a dynamic sequence of a single transaxial slice through the myocardium. This is followed by a discussion of the implication of the technique for analyzing different approaches used in dynamic cardiac SPECT imaging.

\section{THEORY}

Huesman and Mazoyer [7] developed a method for estimating kinetic model parameters from time activity curves for a noisy input function. In their work they developed a formulation for the estimation of both the kinetic parameters and their errors. Most of this work was applied to data in PET applications where the error estimates are calculated from an analytical convolution expression [8]. However in SPECT, because of the need to correct for attenuation in addition to geometric response and scatter, it is necessary to use an iterative reconstruction algorithm and to obtain estimates of the solutions of iterative type algorithms. In this case the error estimates become more difficult to obtain because the inversion of large matrices is required.

In RFIT [9] the kinetic parameters $k_{21}$ (wash-in rate constant), $k_{12}$ (wash-out rate constant), $f_{v}$ (fractional blood volume) of a one-compartment perfusion model are estimated from dynamic cardiac SPECT data by minimizing the chi-square function

$$
\chi^{2}=\rho^{T}(\theta) \Phi^{-1} \rho(\theta),
$$

with respect to the parameters $\theta$. The residuals $\rho$ :

$$
\rho\left(\theta=\left[k_{21}, k_{12}, f_{v}\right]\right)=\beta-H(\theta) \alpha
$$

are the difference between the model and the data. The vector $\beta$ is the reconstructed tissue time activity curve, the vector $\alpha$ is the reconstructed noisy arterial blood input time activity curve, and

$$
H_{i j}= \begin{cases}\int_{t_{j-1}}^{t_{j}}\left(\frac{\tau-t_{j-1}}{t_{j}-t_{j-1}}\right)\left[h\left(t_{i}-\tau\right)-h\left(t_{i-1}-\tau\right)\right] d \tau+\int_{t_{i-1}-t_{j}}^{t_{i}-t_{j}} h(\tau) d \tau & j<i \\ \int_{t_{i-1}}^{t_{i}}\left(\frac{\tau-t_{i-1}}{t_{i}-t_{i-1}}\right) h\left(t_{i}-\tau\right) d \tau & j=i \\ 0 & j>i\end{cases}
$$

where $h(t)=f_{v} \delta(t)+\left(1-f_{v}\right) k_{21} e^{-k_{12} t}$.

In the work presented in this paper we use a weighting matrix, $\Phi=\operatorname{cov}(\rho)$, which provide residual-weighted least squares estimates. For this case $\Phi$ has the expression

$$
\Phi=\operatorname{cov}(\beta, \beta)+H \operatorname{cov}(\alpha, \alpha) H^{T}-H \operatorname{cov}(\alpha, \beta)-\operatorname{cov}(\alpha, \beta)^{T} H^{T} .
$$

Note, that the matrix $\Phi$ is not diagonal, even though the matrices $\operatorname{cov}(\alpha, \alpha), \operatorname{cov}(\alpha, \beta)$, and $\operatorname{cov}(\beta, \beta)$ are assumed to be diagonal. 
In [7] the bias and variance of the estimated parameters were investigated for different weighting matrices $\Phi$. These included $\Phi=\operatorname{cov}(\rho)$ (residual-weighted least squares estimates), $\Phi=\operatorname{cov}(\beta)$ (tissue-weighted least squares estimates), and $\Phi=I$ (unweighted least squares estimates). It was shown that the tissue-weighted and the residualweighted least squares estimate gave almost equal variances, which was much less than the unweighted least squares estimates but that the residual weighted estimates gave the least bias of the three least squares estimates.

To obtain estimate of the covariance matrices $\operatorname{cov}(\alpha, \alpha), \operatorname{cov}(\beta, \beta)$, and $\operatorname{cov}(\alpha, \beta)$ for blood, tissue, and between blood and tissue, respectively, it is necessary to come up with expressions for variances of individual reconstructed pixel values and covariances between reconstructed pixel values. This is developed in the next section.

\section{A. Fixed Point Solution for the Covariance Matrix of the Reconstruction}

Barrett et al. [10] derived an approximate formula for the mean and covariance of the maximum likelihood expectation maximization (ML-EM) reconstruction as a function of iteration number. Wang and Gindi [11] developed a similar result for a MAP-EM reconstruction algorithm. Fessler [12] developed formulations for the mean and the variance at the solution of MAP iterative reconstruction algorithms. Qi [13] presented a more general formulation of the reconstructed errors as a function of iteration. Here we present the fixed-point solution for the covariance matrix of the maximum a posteriori (MAP) reconstruction.

The MAP reconstruction is obtained by maximizing the log of the a posteriori probability function

$$
\begin{aligned}
& \Gamma(f)=\log [p(g \mid f)]-\frac{1}{2} \gamma^{2} f^{T} R f, \\
& \text { subject to } f_{i} \geq 0 \forall i,
\end{aligned}
$$

where $f$ is the image to be reconstructed from projection measurements, $g$ and $-\left(\gamma^{2} / 2\right) f^{T} R f$ is the $\log$ of a Gibbs prior term [14] with parameter $\gamma$ and symmetric positive definite matrix $R$. For the Poisson distribution, we have

$$
\log [p(g \mid f)]=\sum_{m=1}^{M}\left[g_{m} \log \left(\sum_{i=1}^{N} F_{m i} f_{i}\right)-\sum_{i=1}^{N} F_{m i} f_{i}-\log \left(g_{m} !\right)\right],
$$

where $F_{i j}$ are elements of the projection operator $F$. Therefore, writing out $\Gamma(f)$ as a summation, we have

$$
\Gamma(f)=\sum_{m=1}^{M}\left[g_{m} \log \left(\sum_{i=1}^{N} F_{m i} f_{i}\right)-\sum_{i=1}^{N} F_{m i} f_{i}-\log \left(g_{m} !\right)\right]-\frac{1}{2} \gamma^{2} \sum_{i=1}^{N} \sum_{j=1}^{N} R_{i j} f_{i} f_{j} .
$$

The projection operator $F$ models the detector geometry, attenuation, scatter, and the spatially varying geometric response of the collimator. In the simulations presented later, only the effects of photon attenuation and a spatially varying geometric response were modeled for a parallel geometry; but not the effects of scatter.

If one takes the derivative of the penalized log likelihood function $\Gamma(f)$, one obtains

$$
\frac{\partial \Gamma(f)}{\partial f_{l}}=-\sum_{j=1}^{M} F_{j l}+\sum_{j=1}^{M} \frac{g_{j} F_{j l}}{\sum_{i=1}^{N} F_{j i} f_{i}}-\gamma^{2} \sum_{i=1}^{N} R_{l i} f_{i} .
$$

Setting Eq. (8) equal to zero gives the reconstructed image, $\hat{f}(g)$, as long as $\hat{f}>0$. Therefore, we have

$$
S_{l}-\sum_{j=1}^{M} \frac{g_{j} F_{j l}}{\sum_{i=1}^{N} F_{j i} \hat{f}_{i}}+\gamma^{2} \sum_{i=1}^{N} R_{l i} \hat{f}_{i}=0
$$

where $S_{l}=\sum_{j=1}^{M} F_{j l}$ is a sensitivity vector that represents the detection probability for activity in voxel $l$. Note that throughout our development we assume that $\hat{f}$ is positive.

In order to study changes in reconstructed images due to noise in the data we define

$$
\tilde{f} \equiv \hat{f}(\bar{g}),
$$

where $\bar{g}$ is the expectation of the data vector, $g$, so that $\tilde{f}$ is the reconstruction of the noiseless data, we can write

$$
\hat{f}(g)=\hat{f}(\bar{g}+n)=\tilde{f}+\varepsilon,
$$


where $n$ is Poisson noise and $\varepsilon$ is the change in the solution resulting from the noise in the data. Substituting Eq. (11) into Eq. (9) we get

$$
S_{l}-\sum_{j=1}^{M} \frac{\left(\bar{g}_{j}+n_{j}\right) F_{j l}}{\sum_{i=1}^{N} F_{j i}\left(\tilde{f}_{i}+\varepsilon_{i}\right)}+\gamma^{2} \sum_{i=1}^{N} R_{l i}\left(\tilde{f}_{i}+\varepsilon_{i}\right)=0 .
$$

Assuming that the image errors are small compared to the mean, we make the following approximation

$$
\frac{1}{\sum_{i=1}^{N} F_{j i}\left(\tilde{f}_{i}+\varepsilon_{i}\right)} \approx \frac{1}{\sum_{i=1}^{N} F_{j i} \tilde{f}_{i}}\left(1-\frac{\sum_{i=1}^{N} F_{j i} \varepsilon_{i}}{\sum_{i=1}^{N} F_{j i} \tilde{f}_{i}}\right) .
$$

Substituting Eq. (13) into Eq. (12), we obtain the following result:

$$
S_{l}-\sum_{j=1}^{M} \frac{\left(\bar{g}_{j}+n_{j}\right) F_{j l}}{\sum_{i=1}^{N} F_{j i} \tilde{f}_{i}}\left(1-\frac{\sum_{i=1}^{N} F_{j i} \varepsilon_{i}}{\sum_{i=1}^{N} F_{j i} \tilde{f}_{i}}\right)+\gamma^{2} \sum_{i=1}^{N} R_{l i}\left(\tilde{f}_{i}+\varepsilon_{i}\right)=0 .
$$

Separating out the zeroth and first order error terms and dropping the second order error term we have

$$
S_{l}-\sum_{j=1}^{M} \frac{\bar{g}_{j} F_{j l}}{\sum_{i=1}^{N} F_{j i} \tilde{f}_{i}}+\gamma^{2} \sum_{i=1}^{N} R_{l i} \tilde{f}_{i}-\sum_{j=1}^{M} \frac{n_{j} F_{j l}}{\sum_{i=1}^{N} F_{j i} \tilde{f}_{i}}+\sum_{j=1}^{M} \frac{\bar{g}_{j} F_{j l} \sum_{i=1}^{N} F_{j i} \varepsilon_{i}}{\left(\sum_{i=1}^{N} F_{j i} \tilde{f}_{i}\right)^{2}}+\gamma^{2} \sum_{i=1}^{N} R_{l i} \varepsilon_{i}=0 .
$$

The three terms on the left of Eq. (15) sum to zero, as they comprise the left side of Eq. (9) for the solution with noiseless data. The remaining terms can be regrouped to give

$$
\sum_{i=1}^{N}\left[\sum_{j=1}^{M} \frac{\bar{g}_{j} F_{j l} F_{j i}}{\left(\sum_{i=k}^{N} F_{j k} \tilde{f}_{k}\right)^{2}}+\gamma^{2} R_{l i}\right] \varepsilon_{i}=\sum_{j=1}^{M}\left(\frac{F_{j l}}{\sum_{i=1}^{N} F_{j i} \tilde{f}_{i}}\right) n_{j} .
$$

Writing this in matrix notation:

$$
\left(F^{T} \Lambda_{\tilde{g}}^{-1} \Lambda_{\bar{g}} \Lambda_{\tilde{g}}^{-1} F+\gamma^{2} R\right) \varepsilon=F^{T} \Lambda_{\tilde{g}}^{-1} n,
$$

where $\Lambda_{\tilde{g}}$ is a diagonal matrix with the elements of $\tilde{g} \equiv F \tilde{f}$ along the diagonal and $\Lambda_{\bar{g}}$ is a diagonal matrix with the elements of $\bar{g}$ along the diagonal. In the limit of low noise, we see that the error $\varepsilon$ in the image is a linear function of the noise in the data and, thus, the change in the solution resulting from a change in the data is the derivative of the reconstructed image with respect to the data evaluated at the expected data, which is the linear coefficient of the relationship between $\varepsilon$ and $n$ from Eq. (17):

$$
\left.E \equiv \frac{\partial \hat{f}(g)}{\partial g}\right|_{g=\bar{g}}=\left(F^{T} \Lambda_{\tilde{g}}^{-1} \Lambda_{\bar{g}} \Lambda_{\tilde{g}}^{-1} F+\gamma^{2} R\right)^{-1} F^{T} \Lambda_{\tilde{g}}^{-1} .
$$

Eq. (18) is derived by maximizing (with respect to the image distribution $f$ ) the log of the likelihood function for a penalized Poisson distribution in Eq. (7) assuming that $\hat{f}(\bar{g})$ is positive. Also, the assumption in Eq. (13), that the noise is small compared to the mean, is justified in the derivative expression of Eq. (18). It is interesting to note that a Gaussian distribution in Eq. (5) would also give the same expression in Eq. (18) if the same approximations were imposed. The results of our simulation presented later demonstrate that this derivation is sufficiently accurate for a Poisson distribution at the count level appropriate for a dynamic SPECT study.

The covariance matrix for reconstructed values at the solution is given by the approximation [12], [13], [15]

$$
\operatorname{cov}(\hat{f}) \approx E \operatorname{cov}(n) E^{T} .
$$

Since the variance is equal to the mean for a Poisson distribution, the vector of additive projection noise $n$ has the covariance matrix: $\operatorname{cov}(n)=\operatorname{cov}(g)=\Lambda_{\bar{g}}$. This gives us

$$
\operatorname{cov}(\hat{f})=\left(F^{T} \Lambda_{\tilde{g}}^{-1} \Lambda_{\bar{g}} \Lambda_{\tilde{g}}^{-1} F+\gamma^{2} R\right)^{-1} F^{T} \Lambda_{\tilde{g}}^{-1} \Lambda_{\bar{g}} \Lambda_{\tilde{g}}^{-1} F\left(F^{T} \Lambda_{\tilde{g}}^{-1} \Lambda_{\bar{g}} \Lambda_{\tilde{g}}^{-1} F+\gamma^{2} R\right)^{-1} .
$$

If we use the approximation $\tilde{g} \approx \bar{g}$, we get the two expressions:

$$
\begin{aligned}
\operatorname{cov}(\hat{f}) & \approx\left(F^{T} \Lambda_{\tilde{g}}^{-1} F+\gamma^{2} R\right)^{-1} F^{T} \Lambda_{\tilde{g}}^{-1} F\left(F^{T} \Lambda_{\tilde{g}}^{-1} F+\gamma^{2} R\right)^{-1} \\
& \approx\left(F^{T} \Lambda_{\bar{g}}^{-1} F+\gamma^{2} R\right)^{-1} F^{T} \Lambda_{\bar{g}}^{-1} F\left(F^{T} \Lambda_{\bar{g}}^{-1} F+\gamma^{2} R\right)^{-1} .
\end{aligned}
$$

The expression given here for $\operatorname{cov}(\hat{f})$ was derived by Fessler [12] using implicit derivatives. 


\section{B. Covariance Matrix of Estimated Kinetic Parameters}

To obtain weighted least estimates of the kinetic parameters we need to calculate $\Phi$ in Eq. (4). The tissue time activity curve $\beta$ (vector in time) and the noisy arterial blood input time activity curve $\alpha$ (vector in time) at each time frame involve sums of reconstructed counts in the blood and tissue regions of interest:

$$
\begin{aligned}
\alpha^{k} & =\sum_{j \in \text { blood }} \hat{f}_{j}^{k}, \\
\beta^{k} & =\sum_{j \in \text { tissue }} \hat{f}_{j}^{k} .
\end{aligned}
$$

The variances for these regions at each time frame are obtained by appropriately summing the variances of each reconstructed pixel value and covariances between pixel values in the region of interest. This gives the following expressions for the covariance matrices for the time activity curves

$$
\begin{aligned}
& \operatorname{cov}\left(\alpha^{k}, \alpha^{l}\right)=\sum_{j \in \text { blood }} \sum_{j^{\prime} \in \text { blood }} \operatorname{cov}\left(\hat{f}_{j}^{k}, \hat{f}_{j^{\prime}}^{l}\right)=\delta_{k l} \sum_{j \in \text { blood }} \sum_{j^{\prime} \in \text { blood }} \sum_{m=1}^{M} E_{j m}^{k} E_{j^{\prime} m}^{l} \bar{g}_{m}^{k}, \\
& \operatorname{cov}\left(\alpha^{k}, \beta^{l}\right)=\sum_{j \in \text { blood }} \sum_{j^{\prime} \in \text { tissue }} \operatorname{cov}\left(\hat{f}_{j}^{k}, \hat{f}_{j^{\prime}}^{l}\right)=\delta_{k l} \sum_{j \in \text { blood }} \sum_{j^{\prime} \in \text { tissue }} \sum_{m=1}^{M} E_{j m}^{k} E_{j^{\prime} m}^{l} \bar{g}_{m}^{k}, \\
& \operatorname{cov}\left(\beta^{k}, \beta^{l}\right)=\sum_{j \in \text { tissue }} \sum_{j^{\prime} \in \text { tissue }} \operatorname{cov}\left(\hat{f}_{j}^{k}, \hat{f}_{j^{\prime}}^{l}\right)=\delta_{k l} \sum_{j \in \text { tissue }} \sum_{j^{\prime} \in \text { tissue }} \sum_{m=1}^{M} E_{j m}^{k} E_{j^{\prime} m}^{l} \bar{g}_{m}^{k} .
\end{aligned}
$$

These covariance matrices are diagonal since it is assumed the reconstructed values are statistically independent from time frame to time frame. The vectors $\bar{g}^{k}$ are the expected values of the projections measured at the $k$ th time frame, where there are a total of $M$ projection bins and $K$ time frames. The symbol $\delta_{k l}$ indicates that all elements of the matrix is zero except along the diagonal, where $k=l$. Expressions for the derivative matrices $E^{k}$ at time frame $k$ are given in Eq. (18).

The errors in the estimated parameters $\hat{\theta}$ obtained by minimizing Eq. (1) are elements of the matrix

$$
\operatorname{cov}(\hat{\theta})=\Psi
$$

where

$$
\Psi_{i j}^{-1}=\left.\frac{1}{2} \frac{\partial^{2} \chi^{2}}{\partial \theta_{i} \partial \theta_{j}}\right|_{\theta_{i}=\hat{\theta}_{i}, \theta_{j}=\hat{\theta_{j}}} .
$$

This is the asymptotic covariance estimate [15] similar to that specified for the reconstructed values in Eq. (19). For dynamic cardiac SPECT, we have

$$
\Psi^{-1}=\frac{1}{2}\left(\begin{array}{ccc}
\frac{\partial^{2} \chi^{2}}{\partial^{2} k_{21}} & \frac{\partial^{2} \chi^{2}}{\partial k_{21} \partial k_{12}} & \frac{\partial^{2} \chi^{2}}{\partial k_{21} \partial f_{v}} \\
\frac{\partial^{2} \chi^{2}}{\partial k_{12} \partial k_{21}} & \frac{\partial^{2} \chi^{2}}{\partial^{2} k_{12}} & \frac{\partial^{2} \chi^{2}}{\partial k_{12} \partial f_{v}} \\
\frac{\partial^{2} \chi^{2}}{\partial f_{v} \partial k_{21}} & \frac{\partial^{2} \chi^{2}}{\partial f_{v} \partial k_{12}} & \frac{\partial^{2} \chi^{2}}{\partial^{2} f_{v}}
\end{array}\right)_{k_{21}=\hat{k}_{21}, k_{12}=\hat{k}_{12}, f_{v}=\hat{f}_{v}} .
$$

Later the appropriateness of this expression is investigated for the noise levels experienced in dynamic cardiac SPECT.

The explicit expression for $\chi^{2}$ is given by

$$
\chi^{2}=\sum_{k=1}^{K} \sum_{l=1}^{K} \beta^{k} \Phi_{k l}^{-1} \beta^{l}-2 \sum_{k=1}^{K} \sum_{l=1}^{K} \sum_{t=1}^{l} \beta^{l} \Phi_{l k}^{-1} H_{k t} \alpha^{t}+\sum_{k=1}^{K} \sum_{l=1}^{K} \sum_{t=1}^{l} \sum_{t^{\prime}=1}^{k} H_{l t} \alpha^{t} \Phi_{l k}^{-1} H_{k t^{\prime}} \alpha^{t^{\prime}}
$$


where the matrix $\Phi$, which is not diagonal, is given by

$$
\begin{aligned}
\Phi_{k l}= & \delta_{k l} \sum_{j \in \text { tissue }} \sum_{j^{\prime} \in \text { tissue }} \sum_{m=1}^{M} E_{j m}^{k} E_{j^{\prime} m}^{l} \bar{g}_{m}^{k} \\
& +\sum_{j \in \text { blood }} \sum_{j^{\prime} \in \text { blood }} \sum_{m=1}^{M} \sum_{t=1}^{\min (k, l)} H_{k t} H_{l t} E_{j m}^{t} E_{j^{\prime} m}^{t} \bar{g}_{m}^{t} \\
& -\sum_{j \in \text { blood }} \sum_{j^{\prime} \in \text { tissue }} \sum_{m=1}^{M} H_{k l} E_{j m}^{l} E_{j^{\prime} m}^{l} \bar{g}_{m}^{l} \\
& -\sum_{j \in \text { blood }} \sum_{j^{\prime} \in \text { tissue }} \sum_{m=1}^{M} H_{l k} E_{j m}^{k} E_{j^{\prime} m}^{k} \bar{g}_{m}^{k} .
\end{aligned}
$$

The second order partial derivatives of $\chi^{2}$ with respect to the parameters are calculated numerically in our fitting program RFIT [9].

\section{Computer Simulations}

\section{A. Methods}

A dynamic version of the MCAT phantom in Fig. 1 was created for the simulations. Only a single 2D slice of the phantom was used for the experiment. The dynamic phantom was constructed by summing each organ of the static phantom using weights derived from the time-activity curves (TACs) shown in Fig. 2 for each time frame. In this way a series of $2 \mathrm{D}$ phantoms was constructed, one for each time frame of the dynamic acquisition. Examples of activity images for selected time frames are shown in Fig. 3.

The time activity curves shown in Fig. 2 were generated to mimic those seen in patient studies. The myocardium TAC was constructed with blood as input function, with wash-in rate constant $k_{21}=0.824 \mathrm{~min}^{-1}$, wash-out rate constant $k_{12}=0.150 \mathrm{~min}^{-1}$, and blood fraction $f_{v}=0.150$. The background TAC was assumed to be $20 \%$ of the blood TAC.

Projection data were formed by projecting each time frame of the $64 \times 64$ pixel dynamic phantom. The pixels were $7 \mathrm{~mm} \times 7 \mathrm{~mm}$, and the projection bins were $7 \mathrm{~mm}$. The simulated projections included the effects of attenuation and geometric point response, but not scatter. The point response was calculated via ray casting to correspond to a parallel-hole collimator hexagonal holes of $2 \mathrm{~mm}$ diameter and $4 \mathrm{~cm}$ length with an offset of $1 \mathrm{~cm}$ from the detector. For each time frame in the dynamic sequence there were 120 projection angles with each projection containing 64 projection bins.

The dynamic sequence consisted of complete tomographic projections formed at 12 intervals of $5 \mathrm{~s}$, followed by 12 intervals of 10s, followed by 8 intervals of 30s followed by 8 intervals of 60s. Five-second intervals were selected for the initial phase of the dynamic simulation to sufficiently sample the rapid rise in the blood curve. After a minute, which was the peak of the blood curve, the rate of decrease was approximately half the rate of the rise of the blood curve. Therefore, at one minute the sampling interval was increased to $10 \mathrm{~s}$ until three minutes when the interval was increased to 30s. The sampling intervals were increased from $10 \mathrm{~s}$ to $30 \mathrm{~s}$ then from $30 \mathrm{~s}$ to $60 \mathrm{~s}$ in accordance with the decrease in the rate of change in the blood and tissue curves. Each dynamic projection data set was numerically integrated over the active acquisition time for each time frame.

Dynamic sequences were reconstructed using weighted least squares by evaluating $f=\left[F^{T} \Lambda_{\bar{g}}^{-1} F\right]^{-1} F^{T} \Lambda_{\bar{g}}^{-1} g$ in MATLAB, and by maximizing $\Gamma(f)$. A precondition conjugate gradient algorithm [16], [17] was used to maximize $\Gamma(f)$ and it was assumed to converge if the relative change was below $10^{-6}$. This was accomplished usually in about 100 iterations. The projector $F$ and backprojector $F^{T}$ was ray-driven with line length weighting that modeled attenuation and geometric response of the collimator as implemented in [18]. The matrix $R$ in Eq. (5) was defined such that when applied to $f$ it provides a smoothing by penalizing the high frequency components of $f$ in Eq. (5)

$$
f^{T} R f=\sum_{i} \sum_{j \in N_{i}, j>i} R_{i j}\left(f_{i}-f_{j}\right)^{2}
$$

where $R_{i j}$ equals to the reciprocal of the distance between pixel $i$ and pixel $j$, and $N_{i}$ contains the eight nearest neighboring pixels around pixel $i$. 


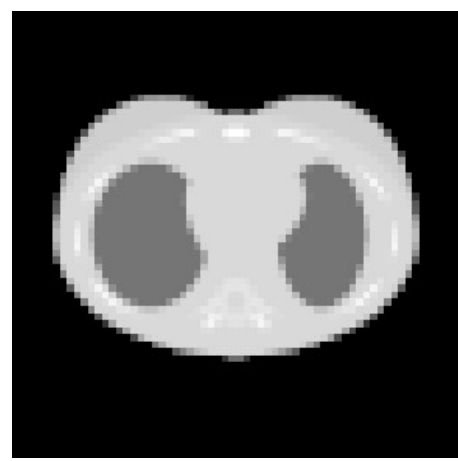

(a)

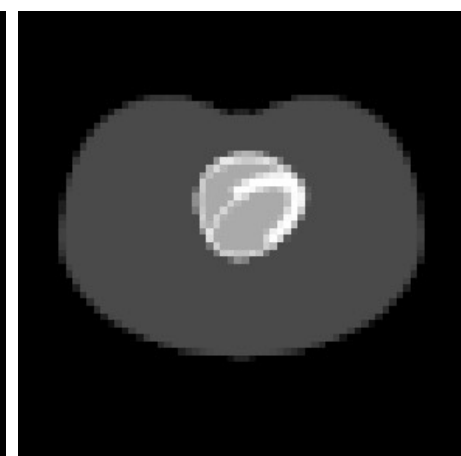

(b)

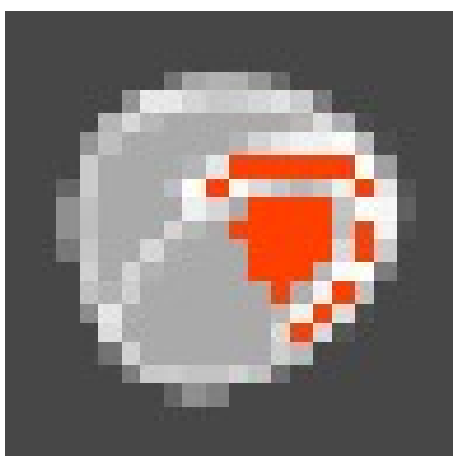

(c)

Fig. 1. A 2D slice of the MCAT phantom used in the simulations: (a) attenuator, (b) emission distribution of blood pool, myocardium, and background, and (c) ROIs in blood and myocardium used in the analysis. The ROIs are highlighted as the darker region.

The elements of $R$ in each column can be represented by the $3 \times 3$ image kernel:

$\begin{array}{ccc}-\sqrt{2} / 2 & -1 & -\sqrt{2} / 2 \\ -1 & 4+2 \sqrt{2} & -1 \\ -\sqrt{2} / 2 & -1 & -\sqrt{2} / 2\end{array}$.

This kernel acts very much like a Laplacian which tends to preserve edges while eliminating noise spikes.

Several values of the parameter $\gamma^{2}$ were tried. The value of 10 was selected for the reconstruction of the 24th frame (the final $10 \mathrm{sec}$ frame) and the value for each other frame was scaled to be inversely proportional to the counts in the frame compared to the counts in the 24th frame. This was done to keep the weighting between the prior and the likelihood function relatively the same from time frame to time frame as the counts change in the data due to the simulated wash-in and wash-out of teboroxime from the myocardium. The value of 10 was selected because it gave reasonable images. The value of the parameter $\gamma^{2}$ did not seem to have a significant effect on the kinetic parameter estimation in our simulations.

Time activity curves were generated from the dynamic reconstructed sequence for the blood region and myocardium region shown in Fig. 1(c). The variance for pixels within the blood and the variance for pixels within the myocardial region and covariance between blood and myocardium pixels were calculated with MATLAB using the expression for $\operatorname{cov}(\hat{f})$ given in Eq. (19). The variances for the blood and myocardial ROIs and the covariance between the blood and myocardial ROI were calculated by appropriately summing the variances and covariances between pixels in the corresponding ROIs. These data were submitted to RFIT to obtain the weighted least squares fit for the kinetic parameters.

\section{B. Results}

Figure 3 shows the results for the weighted least squares reconstructions $\left(f=\left[F^{T} \Lambda_{\bar{g}}^{-1} F\right]^{-1} F^{T} \Lambda_{\bar{g}}^{-1} g\right)$ and MAP reconstructions obtained by maximizing $\Gamma(f)$ with and without noise. It is obvious from the results that the MAP reconstruction considerably suppresses the noise. Also, the MAP reconstruction without noise shows undershoots around the edge of the myocardium due to the formulation of the prior as a $3 \times 3$ smoothing kernel.

Figure 4 shows the variance time sequences for the blood [Eq. (24)] myocardial ROIs [Eq. (26)] time sequence for the negative of the covariance between blood and myocardial ROIs [Eq. (25)]. The discontinuities in the curves are the result of changes in the length of the acquisition interval. In Fig. 4 the correlation is mostly due to the finite system resolution and the smoothing prior. While the variances change significantly over time, the correlation coefficient remains nearly constant. Because blood fraction is a parameter in the kinetic model, the correlation between blood and myocardium does not introduce any bias in the parameter estimation.

Figure 5 and Figure 6 give the results of the fit for the time sequences generated from the Bayesian reconstruction without noise and with noise, respectively. The background curve (lowest curve) illustrates that the reconstructed values match well with the simulated data. The background region in Fig. 1(b) is all pixels in the tissue cross section excluding the central myocardial blood and tissue regions. 


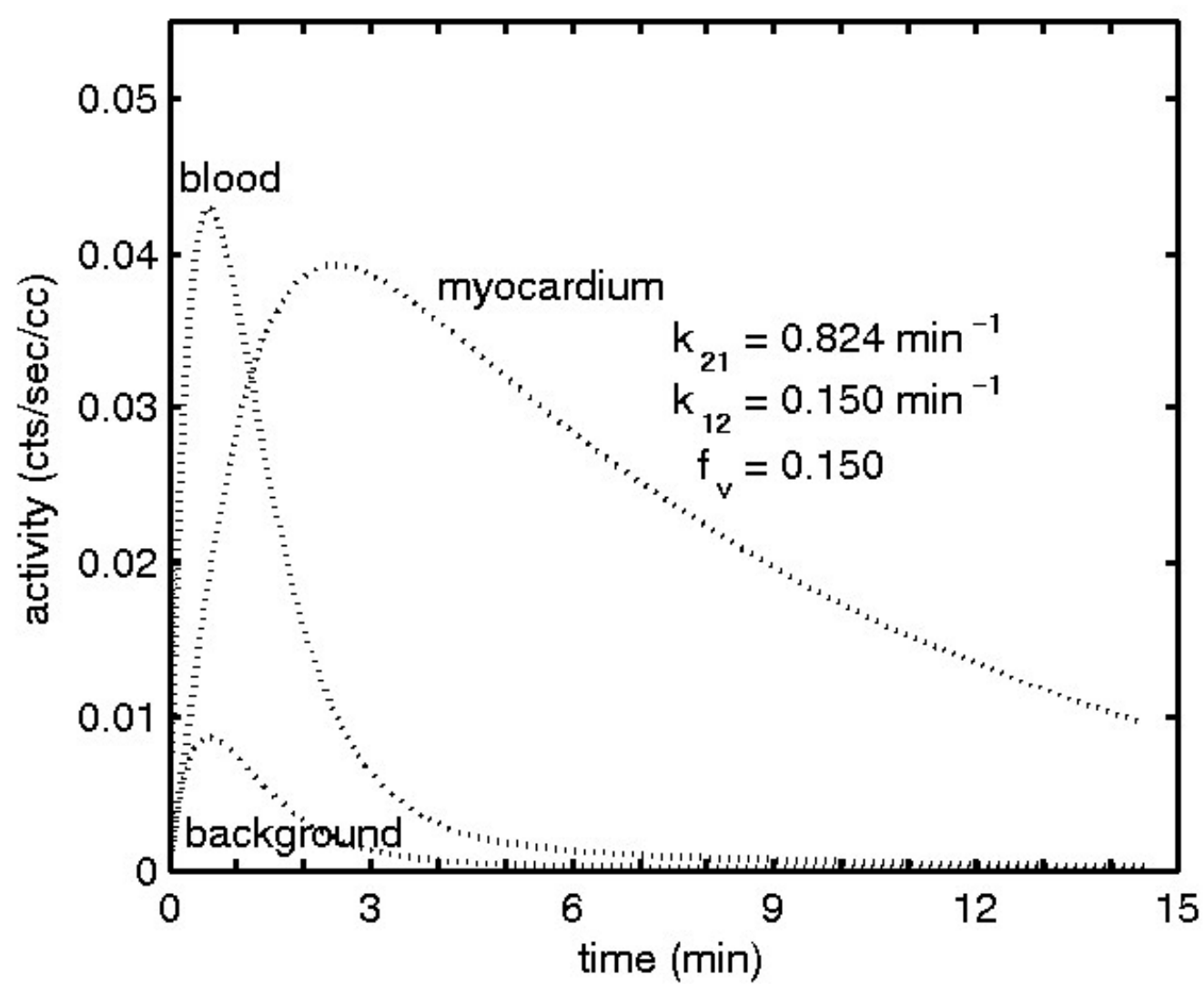

Fig. 2. Time-activity curves used in the simulations: blood input function, extravascular myocardial tissue, and background tissue. The myocardial curve is based on a one-compartment model with a wash-in rate constant $k_{21}=0.824 \mathrm{~min}^{-1}$, wash-out rate constant $k_{12}=0.0150 \mathrm{~min}^{-1}$, and blood fraction $f_{v}=0.150$.

Regularization causes a blurring of blood into the tissue. This is reflected in the increase in $f_{v}$ from the simulated value of 0.150 . The blood fraction increased, thus the tissue fraction has decreased which is reflected by a decrease in the myocardium curves in Figs. 5 and 6. Notice also because of the negative correlation between blood and tissue regions, a positive noise increase above the fitted curve corresponds to a negative variation in the tissue curve and visa versa.

Our previous work [3] gave estimated values and errors for $k_{21}$ of $0.801 \pm 0.325$ using weighted least squares (WLS) and of $0.801 \pm 0.370$ using least squares (LS) when no physical effects were simulated or modeled in the reconstruction; and $0.801 \pm 0.701$ using WLS, and of $0.801 \pm 0.721$ using LS when attenuation, detector response, and scatter were simulated and modeled in the reconstruction. Two things we notice: first, with increase modeling the variance increases, and second, the iterative Bayesian reconstruction provides significant reduction in variance (see Figs. 5 and 6).

\section{DISCUSSION}

We have shown the feasibility of estimating the covariance of time activity curves for MAP reconstruction algorithms, the ability to obtain weighted least squares estimates of kinetic parameters, and the ability to estimate uncertainty of the kinetic parameters. The calculation of $\Phi$ in $\chi^{2}$ [Eq. (1)] requires the calculation of the matrix $E$ [Eq. (18)] for each dynamic reconstruction in the dynamic time sequence. For dynamic cardiac SPECT this can require the inverse of several $4096 \times 4096$ matrices. Our original 40 matrix inversions by SVD required 2 CPU hours on a Cray [3]. Now matrix inversion can be computed in 1 min using row and column operations in MATLAB on a SGI computer $(250 \mathrm{Mhz})$ by reconstructing only nonzero elements. Therefore, efficient estimates of the tissue kinetic parameters in a dynamic cardiac SPECT study can be obtained with present-day desktop computers.

We found that the prior needed to be properly weighted as the counts changed from image to image. Using a fixed weighting parameter $\gamma^{2}$ caused resolution changes from time frame to time frame resulting in changes in 


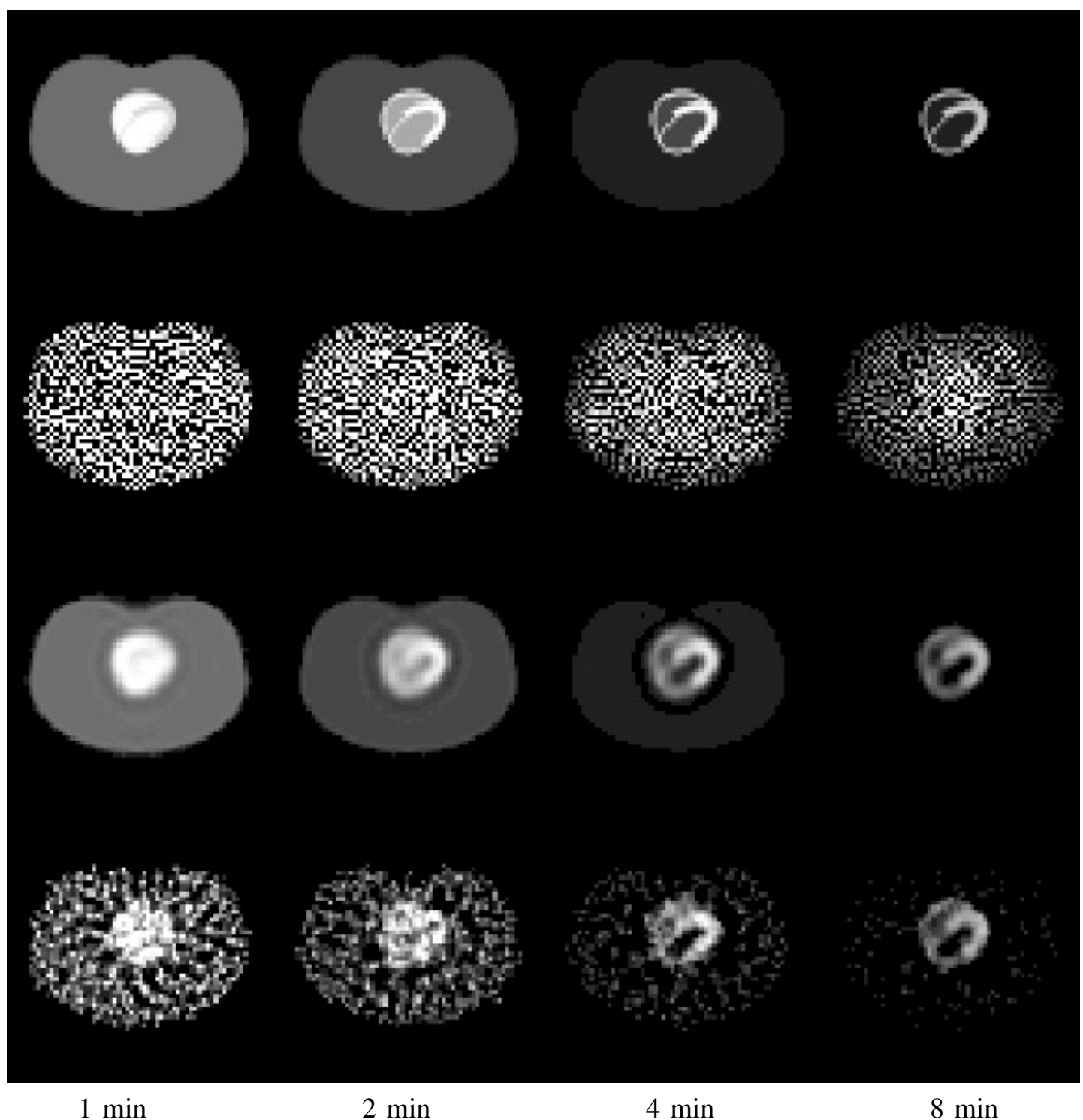

Fig. 3. Sampled Reconstructed Time Sequences. The top row is weighted least squares. The second row is weighted least squares with noise. The third row is Bayesian reconstruction (100 iterations) without noise. The last row is Bayesian reconstruction with noise.

the fractional blood volume $f_{v}$ from frame to frame. The approach taken was to keep the derivative in Eq. (8) relatively constant frame to frame. Comparing the first and second term in Eq. (8) one sees that the first term is relatively independent of the counts, while the second term varies linearly with the counts in the image. Therefore the parameter $\gamma^{2}$ was selected to be equal to 10 for the 24th frame. For the other frames $\gamma^{2}$ was scaled to be inversely proportional to the counts in the frame compared to the counts in the 24th frame so that the two terms in Eq. (8) would be of relatively equal weight as the counts change from time frame to time frame.

This is an approximate solution to the problem. Others have developed more sophisticated methods to handle object dependent resolution [19], [20]. In [19] Qi and Leahy used a spatially variant gamma to achieve uniform contrast recovery. In [20] Stayman and Fessler, designed anisotropic penalty functions for each pixel location to achieve isotropic impulse response function. Future work requires the investigation of these approaches to the application of dynamic cardiac SPECT.

The expression in Eq. (18) is here derived using the fixed-point approach allowing the iteration of the errors to go to convergence. Fessler [12] derived this same result using implicit derivative. By examination of Eq. (18) one can see that the inverse term is the second derivative of gamma with respect to the data whereas the outer term is the first derivative of gamma with respect to the data and the second derivative with respect to the parameter. 


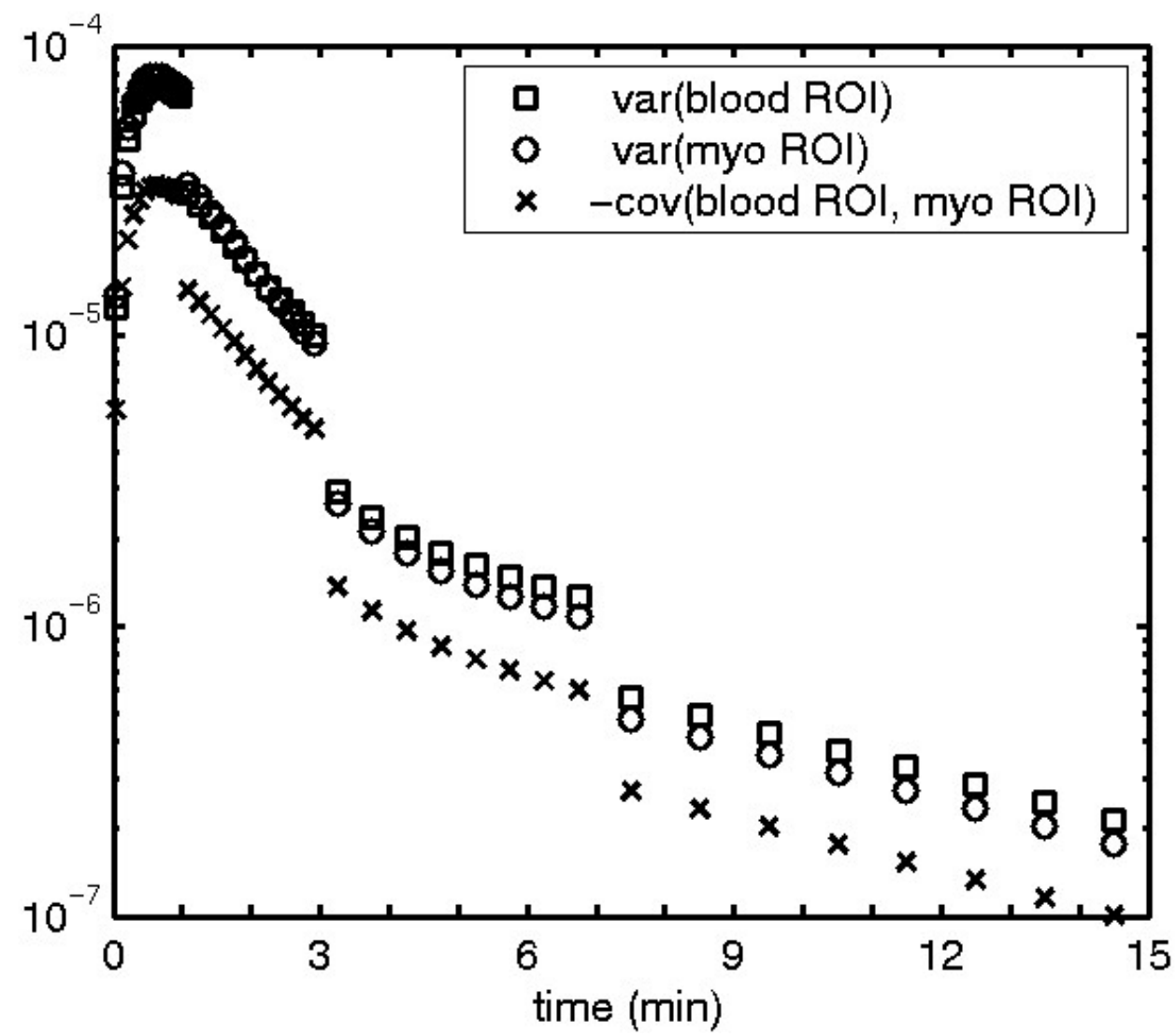

Fig. 4. Variance time sequences for the blood and myocardial ROIs shown in Fig. 1(c) and time sequence for the covariance between blood and myocardial ROIs. These are based on the theory given in Eq. (24), Eq. (26), and Eq. (25). The discontinuities in the curves are the result of changes in the acquisition interval. Note, the negative of the covariance is shown in this plot.

As showed in Fesslers work the same result in Eq. (19) follows naturally by taking the covarianace of the Taylor series approximation of the reconstruction parameter $f$ as a function of the data.

The optimization problem in Eq. (5) requires that the image values be nonnegative. Throughout our development we assumed that the image values were positive so that we could obtain an analytical relationship for the error matrix in Eq. (18). However, to be absolutely correct we need to consider the more general case of the possibility of $f$ being zero. Therefore, the expression for $E$ in Eq. (18) will be inaccurate in low count regions such as intraventricular regions at later time points. There is still the need to develop a more general formulation for $E$ with non negativity constraints.

The methods developed will allow us to better evaluate methodology for dynamic cardiac SPECT imaging. The effects of physiology and physics of the imaging detection process (input function shape, tissue response, statistics, timing resolution, attenuation, scatter, geometric response) on the bias and variance of kinetic parameters can be analyzed at the solution of iterative reconstruction algorithms [21]. Also, the effect of regularization on the bias and variance of the kinetic parameters can better be evaluated.

\section{REFERENCES}

[1] G. T. Gullberg, R. H. Huesman, G. L. Zeng, and S. A. Foresti, "Efficient estimation of dynamic cardiac SPECT kinetic parameters using weighted least squares estimates of dynamic reconstructions," in Conference Record of the 1995 IEEE Nuclear Science Symposium and Medical Imaging Conference, San Francisco, CA, vol. 3, 1995, pp. 1684-1688.

[2] G. T. Gullberg, R. H. Huesman, S. G. Ross, E. V. R. Di Bella, G. L. Zeng, B. W. Reutter, P. E. Christian, and S. A. Foresti, "Dynamic cardiac single-photon emission computed tomography," in Nuclear Cardiology: State of the Art and Future Directions, 2nd ed., B. L. Zaret and G. A. Beller, Eds. St Louis, MO: Mosby, 1999, ch. 11, pp. 137-187.

[3] D. J. Kadrmas, E. V. R. Di Bella, R. H. Huesman, and G. T. Gullberg, "Analytical propagation of errors in dynamic SPECT: Estimators, degrading factors, bias and noise," Phys Med Biol, vol. 44, no. 8, pp. 1997-2014, 1999. 


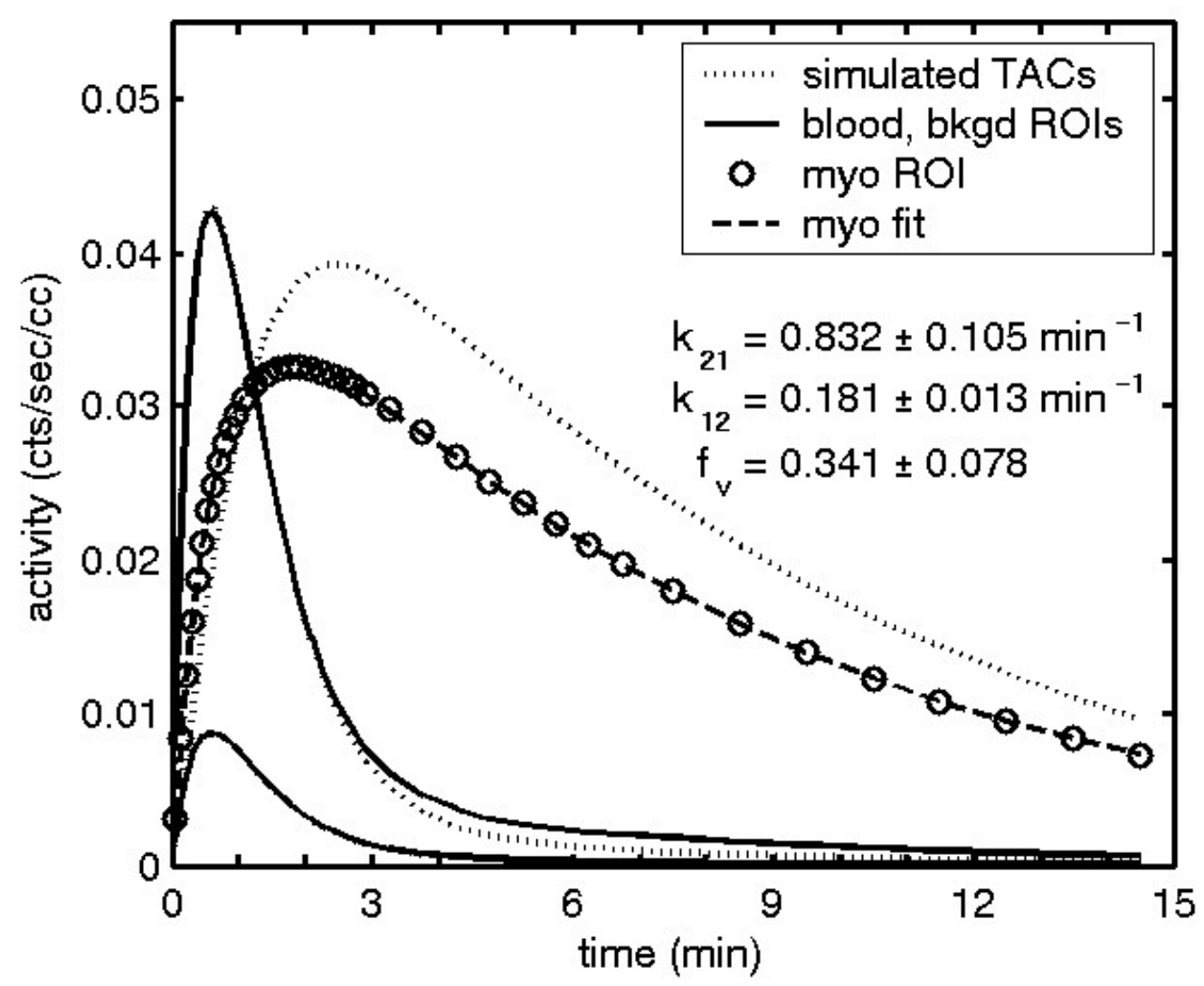

Fig. 5. Fit for the time sequences generated from the Bayesian reconstruction without noise.

[4] K. Lange and R. Carson, "EM reconstruction algorithms for emission and transmission tomography," J Comput Assist Tomogr, vol. 8, no. 2, pp. 306-316, 1984.

[5] E. Levitan and G. T. Herman, "A maximum a posteriori probability expectation maximization algorithm for image reconstruction in emission tomography," IEEE Trans Med Imag, vol. MI-6, no. 3, pp. 185-192, September 1987.

[6] G. T. Gullberg, R. H. Huesman, D. N. G. Roy, J. Qi, and B. W. Reutter, "Estimation of the parameter covariance matrix for a onecompartment cardiac perfusion model estimated from a dynamic sequence reconstructed using map iterative reconstruction algorithms," 2003 IEEE Nuclear Science Symposium and Medical Imaging Conference Record, October 19-25, 2003, Portland, Oregon, pages 3019 $-3023$.

[7] R. H. Huesman and B. M. Mazoyer, "Kinetic data analysis with a noisy input function," Phys Med Biol, vol. 32, no. 12, pp. 1569-1579, 1987.

[8] R. H. Huesman, "A new fast algorithm for the evaluation of regions of interest and statistical uncertainty in computed tomography," Phys Med Biol, vol. 29, no. 5, pp. 543-552, 1984.

[9] R. H. Huesman, B. L. Knittel, B. M. Mazoyer, P. G. Coxson, E. M. Salmeron, G. J. Klein, B. W. Reutter, and T. F. Budinger, "Notes on RFIT: A program for fitting compartmental models to region-of-interest dynamic emission tomographic data," Lawrence Berkeley Laboratory, Report LBL-37621, 1993.

[10] H. H. Barrett, D. W. Wilson, and B. M. W. Tsui, "Noise properties of the EM algorithm: I. Theory," Phys Med Biol, vol. 39, pp. 833-846, 1994.

[11] W. Wang and G. Gindi, "Noise analysis of MAP-EM algorithms for ECT," Phys Med Biol, vol. 42, pp. $2215-2232,1997$.

[12] J. A. Fessler, "Mean and variance of implicitly defined biased estimators (such as penalized maximum likelihood): Applications to tomography," IEEE Trans Image Processing, vol. 5, no. 3, pp. 493-506, March 1996.

[13] J. Qi, "A unified noise analysis for iterative image estimation," Phys Med Biol, vol. 48, pp. 3505-3519, 2003.

[14] S. Geman and D. Geman, "Stochastic relaxation, Gibbs distributions, and the Bayesian restoration of images," IEEE Transactions on Pattern Analysis and Machine Intelligence, vol. PAMI-6, no. 6, pp. 721-41, November 1984.

[15] C. R. Rao, Linear Statistical Inference and its Applications. New York: Wiley, 1973.

[16] J. Qi, R. M. Leahy, S. R. Cherry, A. Chatziioannou, and T. H. Farquhar, "High-resolution 3D Bayesian image reconstruction using the microPET small-animal scanner," Phys Med Biol, vol. 43, pp. 1001-1013, 1998.

[17] R. M. Leahy and J. Qi, "Statistical approaches in quantitative positron emission tomography," Statistics and Computing, vol. 10, pp. 147-165, 2000.

[18] G. L. Zeng, G. T. Gullberg, B. M. W. Tsui, and J. A. Terry, "Three-dimensional iterative reconstruction algorithms with attenuation and geometric point response correction," IEEE Trans Nucl Sci, vol. 38, no. 2, pp. 693-702, April 1991. 


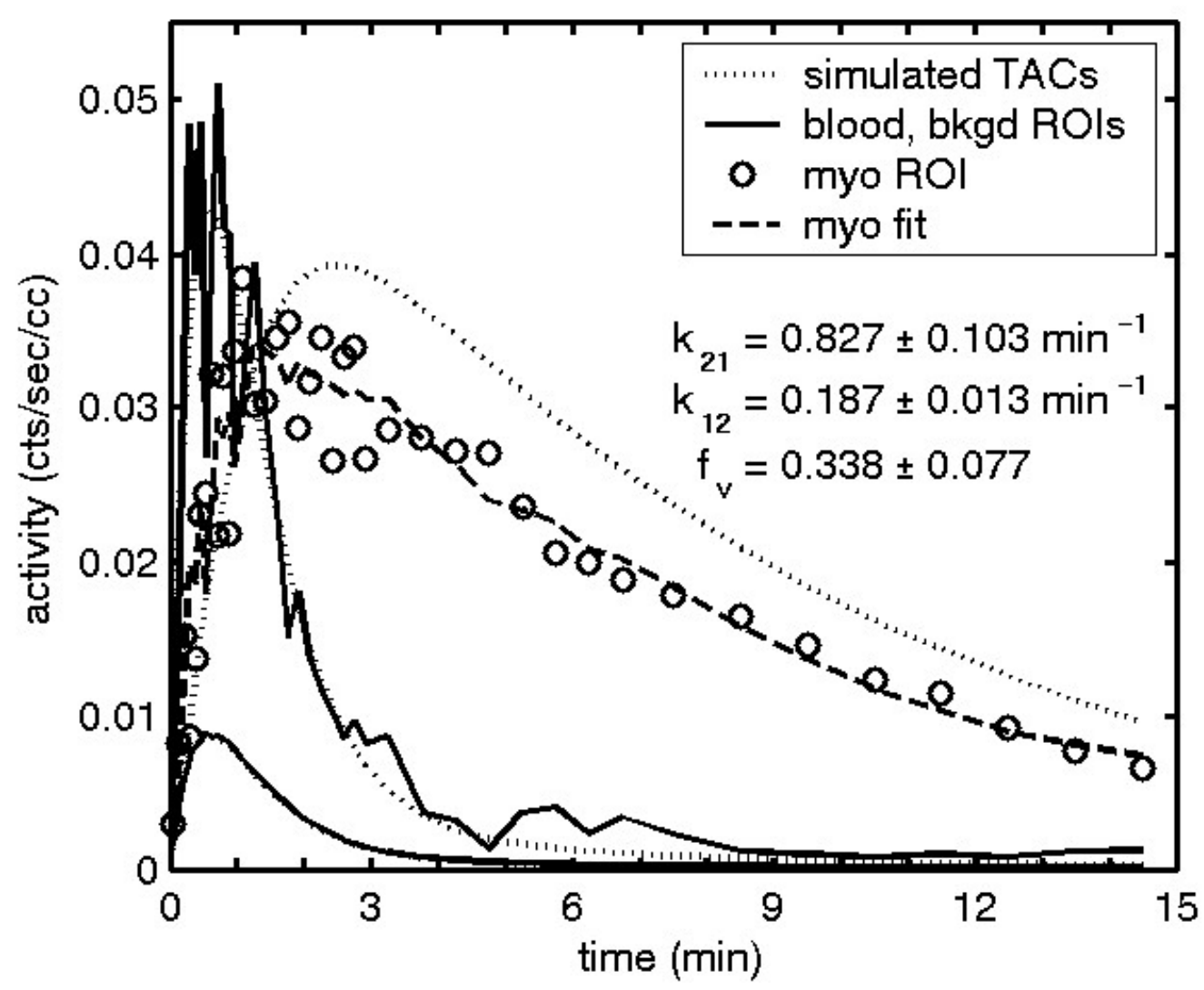

Fig. 6. Fit for the time sequences generated from the Bayesian reconstruction with noise.

[19] J. Qi and R. M. Leahy, "Resolution and noise preperties of MAP reconstruction for fully 3-D PET," IEEE Trans Med Imag, vol. 19, no. 5, pp. 493-506, 2000.

[20] J. W. Stayman and J. A. Fessler, "Regularization for uniform spatial resolution properties in penalized-likelihood image reconstruction," IEEE Trans Med Imag, vol. 19, pp. 601-615, 2000.

[21] S. G. Ross, A. Welch, G. T. Gullberg, and R. H. Huesman, "An investigation into the effect of input function shape and image acquisition interval on estimates of washin for dynamic cardiac SPECT," Phys Med Biol, vol. 42, no. 11, pp. 2193-2213, 1997. 